\title{
La rédactologie : domaine, méthode et compétences
}

\section{Khédija Nakbi}

\section{(2) OpenEdition}

1 Journals

\section{Édition électronique}

URL : http://journals.openedition.org/asp/1428

DOI : 10.4000/asp.1428

ISBN : 978-2-8218-0390-9

ISSN : 2108-6354

\section{Éditeur}

Groupe d'étude et de recherche en anglais de spécialité

Édition imprimée

Date de publication : 30 décembre 2002

Pagination : 15-26

ISSN : 1246-8185

\section{Référence électronique}

Khédija Nakbi, «La rédactologie : domaine, méthode et compétences », ASp [En ligne], 37-38 | 2002, mis en ligne le 15 juillet 2010, consulté le 10 décembre 2020. URL : http://journals.openedition.org/ asp/1428; DOI : https://doi.org/10.4000/asp.1428

Ce document a été généré automatiquement le 10 décembre 2020.

Tous droits réservés 


\title{
La rédactologie : domaine, méthode et compétences
}

\author{
Khédija Nakbi
}

\section{Introduction}

1 Étymologiquement le mot "écrire ", dans de nombreuses et diverses langues, renvoie à l'action des mains qui grattent, gravent, incisent, excavent, évident, coupent, taillent, sculptent ou peignent. Ainsi, par exemple, scribere, en latin, et graphe, en grec, viennent tous deux des racines indo-européennes sker et gerbh, la première signifiant " égratigner ", et la seconde " gratter ", « inciser », de même " to write » en anglais, vient du vieux norrois rîta qui signifie "graver (les runes)»; le même développement sémantique peut être observé, selon I.J. Gelb, dans le groupe des langues sémitiques, le radical $k t b$ « écrire », signifiant originellement « graver » - c'est ce qu'indique le Syriaque maktebà, "poinçon" - et la racine shf signifiant non seulement "écrire » mais aussi " excaver ", « évider » dans les langues sémitiques du sud ; le gothique méljan, «écrire ", signifia d'abord, selon cet auteur, "peindre», comme le montre le mot allemand moderne malen, qui veut dire " peindre » (Gelb $1973: 8$ ).

Mythologie et graphisme multidimensionnel sont d'ailleurs normalement coïncidents dans les sociétés primitives et si j'osais user du strict contenu des mots, je serais tenté d'équilibrer la «mythologie» qui est une construction pluridimensionnelle reposant sur le verbal par une 'mythographie' qui en est le strict correspondant manuel. (Leroi-Gourhan 1964 : 272)

Le même fait est sensible lorsque les Australiens exécutent sur le sable les figures en spirales qui expriment symboliquement le déroulement du mythe du lézard ou de la fourmi à miel, ou lorsque les Aïnous matérialisent dans une coupe de bois sculpté le récit mythisé du sacrifice de l'ours. (273).

2 Ainsi donc, dès l'origine, « écrire » renvoie fondamentalement à différents actes de la vie quotidienne, exercés par l'action des mains sur les choses afin d'y fixer du sens. La science ayant pour objet l'étude des actes de l'écriture est la rédactologie. 


\section{Domaine}

3 Une science se définit essentiellement par son objet, son domaine, sa méthode et l'ensemble des savoirs et savoir-faire qu'elle mobilise. Allant du travail d'écrivain public à celui de webmaster, de l'activité de poète à celle d'ingénieur textuel, de la profession d'enseignant à celle de secrétaire de rédaction, du métier de nègre littéraire à celui de script doctor ou de l'emploi de rewriter à celui d'échotier de chiens écrasés, le domaine de la rédactologie occupe un hyperespace dans lequel on peut distinguer principalement cinq secteurs d'écriture interdépendants et un foyer rédactionnel nodal extrêmement actifs.

\subsection{Secteurs d'écriture}

Les secteurs d'écriture sont essentiellement la linguistique et les sciences du langage, l'enseignement et la recherche scientifique, le livre, les médias et les arts, les nouvelles technologies de l'information et de la communication, ainsi que les divers milieux organisationnels d'exercice des activités de rédaction.

1.1.1. Linguistique et sciences du langage

5 La linguistique et les sciences du langage comprennent des rédacteurs se rapportant les uns aux disciplines centrales de la linguistique, phonétique et phonologie, morphologie et lexicologie, syntaxe, sémantique et pragmatique, ainsi que sémiologie ou sémiotique ; les autres à des disciplines connexes, telles que stylistique, didactique et philologie.

1.1.2. Enseignement et recherche scientifique

6 L'enseignement et la recherche scientifique, lieu de la parole et de l'écriture par excellence, pépinière de rédacteurs, et (avec le fameux adage publish or perish) temple de la rédaction et de la publication, constituent, indubitablement, pour une rédactologie scientifique naissante, un terreau idéal pour son développement.

1.1.3. Livre, médias et arts

7 Le livre, les médias et les arts, dans lesquels travaille une myriade de rédacteurs. Dans le livre, on trouve des " porte-plumes » tels que rédacteur littéraire, rédacteur technique, auteur-écrivain et nègre-écrivain, ghost-writer, rewriter, réviseur et correcteur, documentaliste, etc.

8 Dans le journalisme, écrit, audio et télévisuel, et les métiers de la communication, s'affaire une multitude de spécialistes en écriture, journalistes, rédacteurs, secrétaires de rédaction, pigistes, reporters, éditorialistes, feuilletonistes, chroniqueurs, courriéristes, échotiers, responsables d'édition, attachés de presse, journalistes d'entreprise...

Dans la publicité, il y a les métiers de chef de publicité, directeur de création, directeur artistique, planificateur stratégique, concepteur rédacteur, assistant de rédaction...

10 Dans le cinéma, le théâtre, la musique et la danse, il y a les métiers de scénariste, scripte, compositeur, chorégraphe, etc.

1.1.4. Technologies de l'information et de la communication

11 Les technologies de l'information et de la communication (TIC) ont généré un rédacteur de type nouveau, travaillant, non plus sur un texte, mais sur un hypertexte ou macrotexte 
et dans un univers rédactionnel qui n'est plus physique, mais virtuel : rédacteur sur le Web, webmaster, web designer, développeur/concepteur multimédia, auteur-scénariste, etc.

\subsubsection{Milieux organisationnels}

Les milieux organisationnels comprennent une pléthore de secrétaires, d'employés de bureau, de clercs, d'employés aux écritures, ainsi qu'une cohorte de chargés d'études et de rédacteurs techniques travaillant dans le tertiaire et au sein, notamment, d'entreprises industrielles et de sociétés d'ingénierie.

\subsection{Foyer rédactionnel nodal}

13 La terminologie, la néologie et la traductologie fournissent des rédacteurs dont les fonctions sont essentiellement :

1. pour les terminologues, «d'effectuer le travail terminologique systématique (élaboration des dictionnaires, lexiques et vocabulaires) et ponctuel (décision sur les consultations terminologiques établies par les traducteurs, les rédacteurs et les superviseurs)» (Cabré 1998 : 258) ;

2. pour les néologues, d'une part, en lexicographie, « d'actualiser les dictionnaires de la langue générale ", d'autre part, en terminologie, de "créer ou adapter les dénominations des langues de spécialités », enfin, en planification ou aménagement linguistique, « d'établir des critères qui orientent la création lexicale » (Cabré $1998: 258)$;

3. pour les traducteurs, de transposer d'une langue étrangère de départ dans une langue d'arrivée, sur le mode écrit, en mettant en oeuvre leur maîtrise des langues utilisées, leurs connaissances approfondies des cultures nationales ou organisationnelles, des techniques de traduction écrite, et des savoirs et savoir-faire spécifiques concernant les différents domaines d'intervention choisis. «Pour les traducteurs, la terminologie facilite la traduction d'un contenu d'une langue à une autre » (Cabré 1998 : 93).

14 Terminologie, néologie et traduction font ainsi partie d'un conglomérat dynamique composé par l'ensemble des secteurs d'écriture; ils constituent, au sein de cet hyperespace rédactologique en perpétuel mouvement, un noyau extrêmement actif, alimentant les différents secteurs tout en étant alimenté par eux, dans un processus de transformation et d'enrichissement langagier incessant (cf. Fig. 1). 


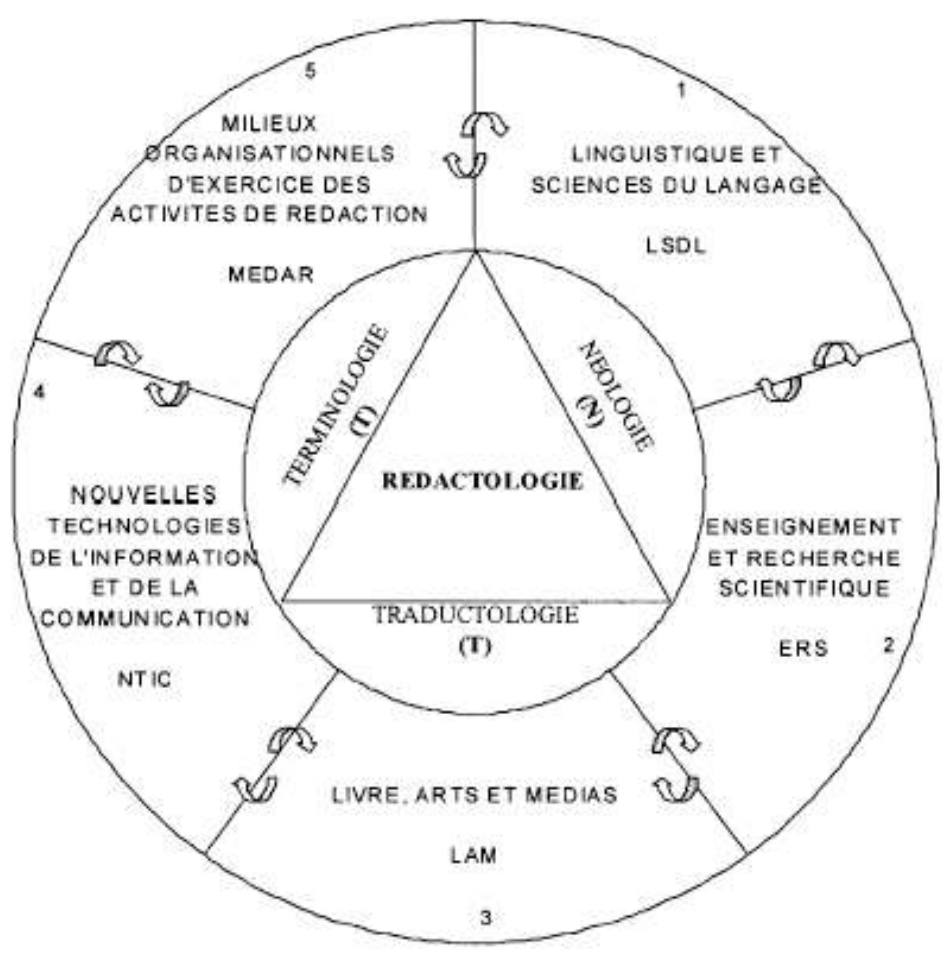

15 Cet inventaire des métiers de rédactologue, quelque peu pléthorique et fastidieux, certes, est cependant loin d'être exhaustif, tant le champ rédactologique est à la fois extraordinairement vaste et varié. Gendelettre, grimaud, gratte-papier, scribouillard, écrivailleur, folliculaire, plumitif ou belle plume, le domaine du rédacteur est univoquement celui des actes de l'écriture. La rédactologie est la science des conduites scripturales.

\section{Méthode}

Du jeu du cadavre exquis des surréalistes, des calligrammes pittoresques d'un Apollinaire, ou du discours intimiste des Confessions d'un Rousseau jusqu'au document technique ou scientifique, de la notice à la thèse en passant par l'affiche publicitaire, du texte à l'hypertexte, le champ méthodologique de la rédactologie, également complexe, se structure selon deux dimensions bipolaires fondamentales.

\subsection{La première dimension}

La première dimension, relative à la forme du document, s'étend sur un axe que l'on pourrait appeler «subnormé vs surnormé » (astreintes normatives plus ou moins fortes en matière de codes de rédaction, de normalisation ISO, AFNOR, MLA, APA).

Au pôle surnormé dominent, pour reprendre les conceptions de R. Jakobson (1963), d'une part la fonction phatique (centration sur le canal), sur le plan de la forme, laquelle se trouve en quelque sorte uniformisée, neutralisée, de manière à faciliter la communication, en permettant au lecteur « une concentration optimale sur le contenu 
textuel » (APA 1990 : 11), et d'autre part la fonction métalinguistique (centration sur le code), sur le plan du vocabulaire, par l'emploi d'une terminologie normalisée, laquelle constitue «le meilleur recours qu'ont les spécialistes pour se référer à un domaine de spécialité dont ils partagent les connaissances à des degrés variés » (Cabré 1998: 93). À cette extrémité de l'axe, l'émotif, l'expressif, le poétique et l'esthétique sont généralement absents (117) ; ici font autorité les termes univoques et les syntagmes plus ou moins figés des répertoires terminologiques spécialisés. « Rien n'est objectif que ce qui est identique pour tous » (Poincaré $1970: 180$ ).

Au pôle subnormé dominent, en revanche, les fonctions poétique, esthétique ou ludique (centration sur le message), et conative (centration sur le récepteur) ; là règnent les mots dans toutes leurs splendeurs lexicales, les images et les symboles avec toutes les ambiguïtés et la surdétermination sémantiques qui les caractérisent. M. Merleau-Ponty écrit : «C'est pour avoir été employé dans différents contextes, que le mot peu à peu se charge d'un sens qu'il n'est pas possible de fixer absolument » (1945: 445).

\subsection{La deuxième dimension}

21 La deuxième dimension est relative au fond et, plus spécialement, à la méthode objective ou subjective adoptée par le rédacteur .

$\mathrm{Au}$ pôle objectif dominent la fonction référentielle (centration sur le référent) et la tendance naturaliste et empiriste fondées essentiellement sur le raisonnement scientifique et la vérification expérimentale. C. Bernard écrit : "Le savant complet, est celui qui embrasse à la fois la théorie et la pratique expérimentale» (1966:54). E. Durkheim affirme : "La première règle est la plus fondamentale, est de considérer les faits sociaux comme des choses » $(1967: 15)$; ici, la réalité n'existe que dans la mesure où elle est expérimentée, mesurée, a posteriori. «Il n'y a pas de réalité préalable que l'on mesure ; il y a une réalité qui naît du moment où elle est mesurée » (Ullmo 1969 : 44).

$\mathrm{Au}$ pôle subjectif dominent, en revanche, la fonction expressive ou émotive (centration sur l'émetteur) et la tendance humaniste, à caractère littéraire et philosophique; là la réalité n'existe (notamment du point de vue phénoménologique et existentiel) que du moment où elle est expériencée, vécue hic et nunc. «Si l'on détache le monde objectif des perspectives finies qui ouvrent sur lui et qu'on le pose en soi, on ne peut y trouver de toutes parts que des 'main-tenants' » (Merleau-Ponty 1945 : 471).

Le croisement de ces deux axes méthodologiques fondamentaux permet de distinguer quatre grands types de rédactologie (voir fig. 2) :

1. scientifique et technique (surnormé/objectif);

2. littéraire et philosophique (surnormé/subjectif) ;

3. poétique, esthétique et ludique (subnormé/subjectif);

4. publicitaire et propagandiste (subnormé/objectif). 
Figure 2. Système méthodologique en rédactologie

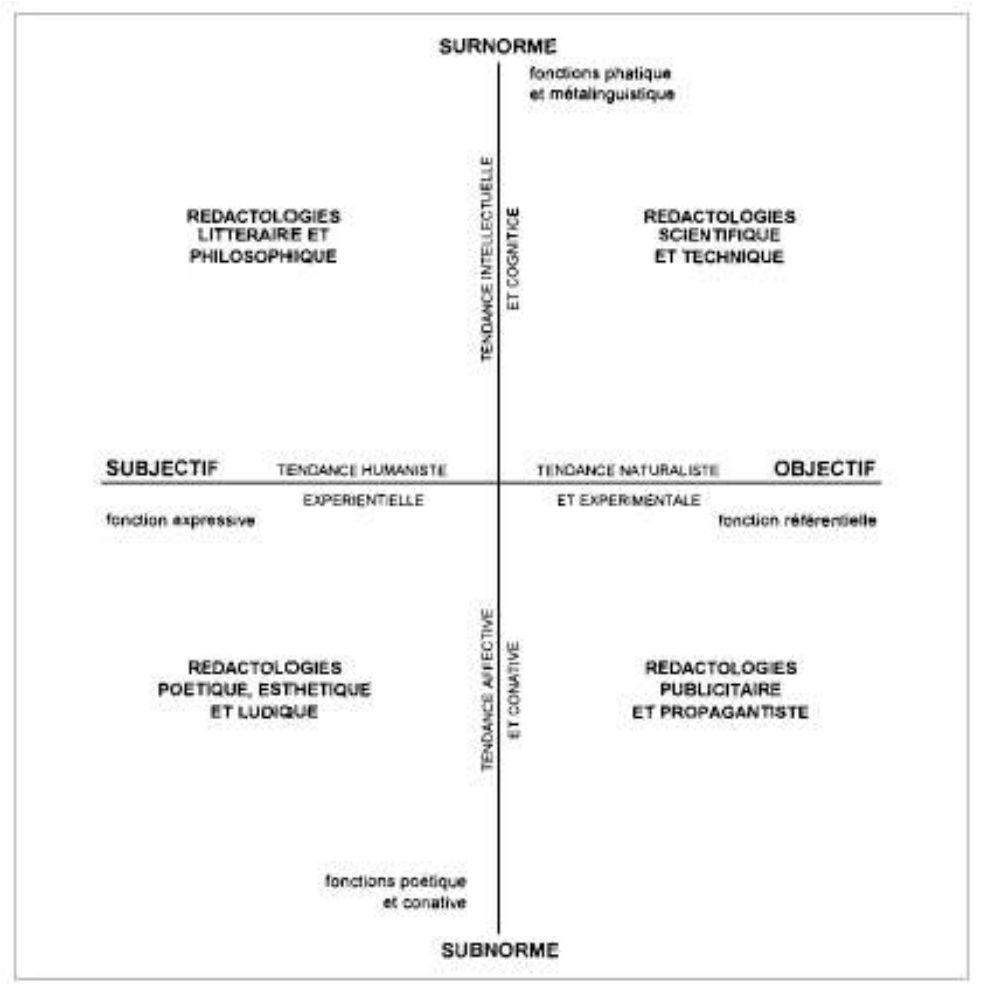

Ainsi se dessinent, en une sorte de coincidentia oppositorum, deux principales orientations méthodologiques, à la fois irréductibles et inséparables l'une de l'autre, avec d'une part, une rédactologie à la troisième personne, référentielle et objective, plus abstraite, et d'autre part, une rédactologie à la première et deuxième personne, essentiellement (inter-) subjective et expérientielle, plus pragmatique et concrète.

\section{Compétences}

\subsection{Définition générale}

Le mot « compétence », apparu au quinzième siècle (Gilbert 1993 : 59), est fort complexe et a donné lieu à une littérature abondante, en France et à l'étranger. Cependant, malgré la multiplicité des points de vue, cette notion fait aujourd'hui l'objet, quant à sa signification générale, d'un certain consensus.

1. La compétence est une variable hypothétique (hypothetical construct) dont on n'observe que les manifestations dans le comportement du sujet agissant.

2. La compétence est inséparable de l'action et ne peut être appréhendée qu'au travers de l'activité par laquelle elle s'exprime et dont elle permet la réalisation; elle est toujours « compétence à agir » dans une situation et un but déterminés.

3. La compétence est un ensemble composite de savoirs, savoir-faire, savoir-être, savoirapprendre et faire-savoir, de "conduites-types, de procédures-standards, de types de raisonnement, que l'on peut mettre en oeuvre sans apprentissage nouveau » (De Montmollin $1984: 122)$. C'est un comportement observable qui «matérialise une ou plusieurs capacités possédées par le sujet » (Le Boterf $1990: 22$ ). 
4. La compétence ne se décrit pas seulement en termes de contenu, mais aussi et surtout en terme de structure, dans la mesure où elle combine de façon dynamique les différents éléments qui la constituent.

5. La compétence produit la performance attendue; elle implique donc, pragmatiquement, les notions de réalisation et d'efficience.

Compte tenu de ces caractéristiques, on peut convenir d'appeler compétence la capacité d'une personne (individu ou groupe) à mobiliser, en fonction d'un but, dans une situation déterminée, un ensemble structuré d'attitudes et d'actions, de manière à répondre de façon pertinente et efficace à la situation.

\subsection{Compétences en rédactologie et traductologie}

\subsubsection{Pluralité et diversité des compétences}

Il n'est pas donc pas surprenant, eu égard à l'étendue de son domaine et à la variété de sa méthode, que la rédactologie, en tant qu'ensemble de savoirs et de savoir-faire inhérents à l'acte d'écrire, requière une nécessaire polyvalence et de très nombreuses compétences. On peut aisément constater cette réalité à la lecture du Répertoire opérationnel des métiers et emplois (ROME) et des «fiches fonctions » de l'Association pour l'emploi des cadres (APEC). Certaines compétences sont simples ou élémentaires, d'aucunes plus complexes; les autres, dites transversales, sont des compétences génériques à une large gamme d'activités et sont, nonobstant l'extrême diversité des secteurs, communes à plusieurs spécialités et réutilisables dans un grand nombre de situations rédactionnelles.

1. Compétences techniques: maîtrise de la langue française et d'une langue étrangère, notamment l'anglais; maîtrise des techniques de communication écrite, des outils bureautiques et multimédias, maîtrise de logiciels de traitement de texte, de tableur, de gestion de bases de données documentaires, de banques de terminologie, d'aide à la traduction, voire de traitement statistique des données.

2. Compétences humaines : telles que clarté, pédagogie, précision, rigueur, ponctualité, curiosité, adaptabilité et créativité...

Les autres compétences du rédacteur, nettement plus nombreuses et plus spécifiques, sont étroitement liées à tel ou tel milieu institutionnel, à tel ou tel environnement organisationnel, à tel ou tel particularisme mésologique, à tel ou tel poste de travail, à telle ou telle spécialité exercée, ou à tel ou tel genre d'écrit.

$\mathrm{Au}$ sein de cet espace de compétences rédactologiques, aussi nombreuses que variées, il est une capacité qui se trouve systématiquement liée au profil du rédacteur et, plus particulièrement à celui du rédacteur-traducteur; il s'agit de la compétence en matière de communication.

\subsubsection{Compétence communicative ou archi-compétence du traducteur}

La notion de compétence selon Noam Chomsky est la capacité à produire et à interpréter des énoncés ; elle se confond en linguistique, dans une acception étroite, avec l'aptitude à fabriquer des phrases grammaticales. En revanche, la compétence communicative, concept élaboré par D.H. Hymes s'inscrit, quant à elle, dans une acception plus large, dans la mesure où $\mathrm{y}$ est prise en compte la dimension relationnelle, les ressources communicatives ne se réduisant pas seulement à un corpus linguistique. 
Cela est d'autant plus évident quand il s'agit de ce cas particulier de la communication qu'est la traduction.

Bien plus, la traduction est un cas remarquable de la communication, c'est une métacommunication; une communication au second degré qui, d'une langue à l'autre, porte sur la communication au premier degré qu'elle prend pour objet. $C^{\prime}$ 'est-à-dire que la traduction procède à une objectivation de la communication en langue-source qu'elle globalise pour en faire le contenu du message qu'elle a à traduire en langue-cible. La méta-communication traduisante fait de la communication-objet au premier degré, en langue-source, un donné sociolinguistique. (Ladmiral $1994: 144$ ) une « archicompétence » (1994: 176), en ce sens qu'elle est susceptible d'intégrer en un tout dynamique la triadisation inhérente à tout processus de traduction, à savoir les trois pôles correspondant à l'auteur, au traducteur et au lecteur, ainsi qu'à leurs interrelations: relations directes, traducteur-texte, traducteur-auteur et traducteurlecteur, et relation indirecte, lecteur-auteur via traducteur. Ces différentes relations déterminent, à notre avis, les trois compétences fondamentales complexes et transverses, dont on peut penser qu'elles sont génériques à toutes les situations de traduction et d'interprétation, à savoir, la compréhension, la compréhensibilité et la crédibilité ( cf. fig. 3)

Figure 3. Structure de l'archi-compétence du traducteur

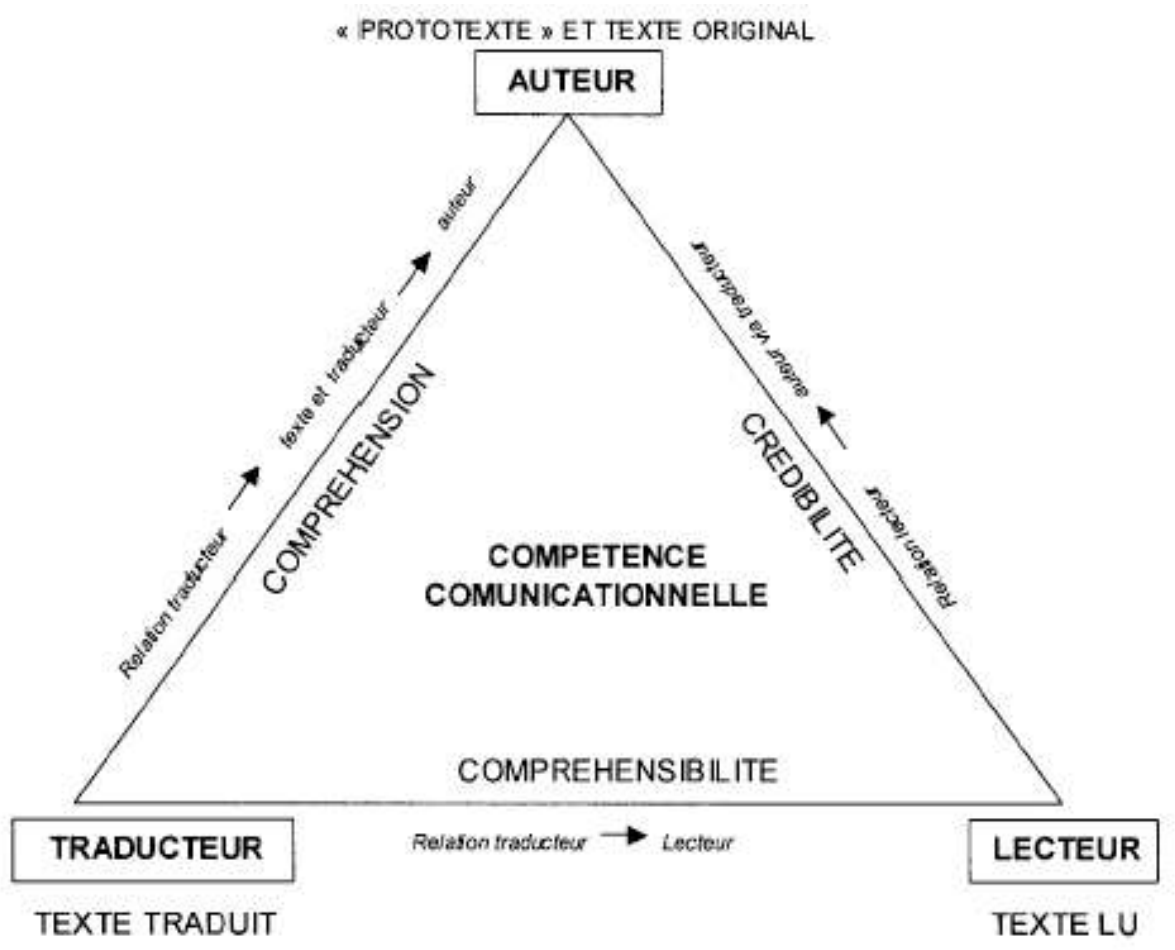

\subsubsection{Compréhension}

Dans tout énoncé, on peut distinguer d'une part, la signification intrinsèque des mots qui le composent, et d'autre part, la signification extrinsèque qui leur est dévolue par le destinateur, d'où, du point de vue du traducteur, deux types ou niveaux de 
compréhension, celle du dire du document écrit à traduire, et celle du vouloir dire de l'auteur, producteur du texte original.

La compréhension de premier niveau correspond à l'appréhension cognitive, en tant que « faculté de saisir par l'intelligence » (Lalande 1968: 72), par le traducteur de la forme et $\mathrm{du}$ fond du texte à traduire. Il s'agit de « la compréhension 'herméneutique' du signifié textuel'l'intelligence du texte' » (Ladmiral 1994 : 63).

Peuvent s'inscrire ici les deux approches de la lecture en langue spécialisée, d'une part l'approche «bottom-up » qui correspond à un processus ascendant du décodage de l'écriture: lettres et mots, syntagmes, phrases, paragraphe, structure de texte, compréhension; d'autre part l'approche "top-down» qui correspond à un processus descendant du décodage du texte : connaissance du monde, connaissance de la spécialité, connaissance du texte, aptitude à la déduction logique, connaissances linguistiques, compréhension.

Ces deux approches «ne doivent pas être mutuellement exclusives mais doivent être envisagées comme recours à tout moment susceptible de fonctionner là où l'autre ne répond pas aux besoins ponctuels » (Resche 1999 : 355). Par ailleurs, un texte n'est jamais un isolat; il doit être perçu comme «activateur ou réactivateur du macrotexte, défini comme 'niveau textuel préconstruit formé non seulement de termes mais de chaînes syntaxiques pré-assertives qui conditionnent la compréhension et la production du discours scientifique dans un domaine donné' » (Magnet 2001 : 54).

La compréhension de deuxième niveau correspond à l'appréhension phénoménologique en tant qu'effort de saisir "directement par intuition» (Lalande 1968: 769) par le traducteur du « vouloir dire » de l'auteur. Là il s'agit non seulement d'être à l'écoute du texte rédigé, mais aussi et surtout de comprendre, " entre les lignes ", aux interstices du discours, ce que l'auteur a voulu dire. C'est ce que nous appelons le « q.q.p.b.v.d.p.l. » du traducteur, à savoir cette lancinante et, parfois même, très angoissante question: " qu'est-ce qu'il (elle) peut bien vouloir dire par là?»

Selon M. Merleau-Ponty, il n'y a pas de compréhension véritable sans que l'on tienne compte de ce qu'il appelle l'intention totale.

Qu'il s'agisse d'une chose perçue, d'un événement historique ou d'une doctrine, « comprendre », c'est ressaisir l'intention totale - non seulement ce qu'ils sont pour la représentation, les "propriétés » de la chose perçue, la poussière des «faits historiques", les «idées" introduites par la doctrine - mais l'unique manière d'exister qui s'exprime dans les propriétés du caillou, du verre ou du morceau de cire, dans tous les faits d'une révolution, dans toutes les pensées d'un philosophe. (Merleau-Ponty 1945 : XIII)

La compréhension, au sens phénoménologique du mot, implique notamment les notions de totalité et de point de vue de l'autre. L'enjeu serait ici, en tentant, autant que faire se peut, de s'introduire empathiquement dans l'univers psychique de l'auteur, de retrouver le sens primitif et primordial du « texte prototypique idéel » ou « prototexte » dont nous subsumons l'existence, et dont le document à traduire ne serait, en somme, qu'une copie ; et ce, afin de reproduire, dans le texte traduit, le sens du texte original dans toute son authenticité.

L'acte de traduire exige donc une symbiose entre l'intention qui a présidé chez l'auteur à l'acte d'expression et l'intention de son alter ego, le traducteur, au moment où ce dernier s'apprête, à son tour, à formuler ce vouloir dire. (Déjean Le Féal $1994: 11$ ) 
Ce qui caractérise la démarche du traducteur par rapport à celle du simple lecteur est donc l'espèce de jusqu'auboutisme avec lequel il remonte la filière du message en partant de sa réalisation langagière jusqu'à l'état où il se trouvait avant toute formulation. (Déjean Le Féal 1994 : 13)

Il convient, cependant, de bien préciser qu'il ne s'agit aucunement de compréhension passive et fusionnelle (traducteur-auteur), comme pourrait le suggérer le mot empathie, équivalent français de l'allemand Einfühlung, mais d'une compréhension active et ouverte, englobant dans une totalité dynamique le moi, l'autre et le monde; il serait alors plus exact de parler non pas de Einfühlung, sorte d'intuition participante, mais de Weltanschauung qui « inclut le Moi dans le monde et fait référence tant aux conceptions de soi qu'à celles du monde extérieur » (Fromaget 1975: 902). Il s'agit donc bien d'une conception du monde, d'une vision partagée, grâce à laquelle la communication entre les êtres, malgré leurs différences, et, notamment, la transposition ou la traduction des discours les uns dans les autres, en dépit de leurs dissimilitudes, deviennent possibles. A. Schaff écrit :

Les différents systèmes linguistiques ne sont pas des systèmes qui ne posséderaient pas un seul élément commun et seraient de ce fait réciproquement impénétrables, intraduisibles. Un problème philosophiquement intéressant consisterait à savoir si des destinées biologiques totalement différentes (en supposant que des êtres pensants seraient découverts sur une planète) produiraient des langues intraduisibles, bien que ces autres systèmes de langage et de pensée seraient également un certain reflet de la réalité. (1969:226)

L'intraduisible relèverait, par conséquent, moins de l'existence d'une incompatibilité essentielle, ou d'une absence d'équivalence partielle entre deux ou plusieurs systèmes de langages, que de la manifestation d'une défaillance individuelle liée davantage au niveau de formation et/ou de compétence du traducteur. «C'est sur une telle archi-compétence tendanciellement totale qu'est obligé de faire fond le traducteur » (Ladmiral 1994 : 177).

Il convient enfin de souligner que ces niveaux de compréhension, relevant tous deux de la linguistique textuelle, se complètent parfaitement, l'un apportant rigueur et sécurité à la démarche de traduction, et l'autre, approfondissement et ouverture. L'analyse textuelle étant un outil, selon C. Durieux, ayant précisément pour fonction de pallier le danger que fait courir la rapidité de l'intuition, et de conférer une certaine sécurité au traducteur dans sa démarche (1997: 52).

\subsubsection{Compréhensibilité}

Cette deuxième compétence-clé a trait essentiellement à la relation traducteur-lecteur ; car traduire c'est simultanément écouter pour comprendre, et « comprendre pour faire comprendre " (Durieux 1997: 49). Pour atteindre efficacement le destinataire, le traducteur doit mobiliser toutes les ressources rédactionnelles et traductionnelles disponibles, en matière notamment de clarté textuelle, de lisibilité et de transparence, de cohésion et de cohérence, de pertinence, de stratégies argumentaires, de procédés de rhétorique et de recours aux différents outils d'aide à la traduction.

La capacité d'énoncer et de dire avec clarté, selon C. Beaudet (2000), ne suit pas la capacité de comprendre : elle l'accompagne, elle s'y ajoute, du fait qu'elle a fait l'objet d'un apprentissage sérieux et spécifique. Plus qu'une retombée du génie de chacun, la clarté serait, dans cette perspective, un effet d'apprentissage et de compétence acquise. 


\subsubsection{Crédibilité}

\section{requises, la rédactologie, science des conduites scripturales, constitue véritablement un} mégasystème dont l'unité est à rechercher dans sa complexité même.

Les métiers de l'écriture en général et, plus spécialement, ceux de la traduction, s'inscrivent dans un processus dynamique dont les principaux axes d'évolution prévisibles pour les années à venir paraissent être les suivants :

1. tertiarisation accrue des professions, en raison notamment du développement des loisirs et du vieillissement de la population ;

2. prééminence du relationnel, de la capacité à communiquer, d'ouverture sur l'extérieur et d'attention aux autres, qui tendent à devenir « presque aussi importants que la formation en elle-même » (Lartigue 1999 : 45) ;

3. nécessité grandissante d'une maîtrise des TIC, exigeant l'acquisition incessante de nouvelles compétences, de la part des travailleurs, et une adaptation continue. Ainsi, par exemple, la Mutuelle complémentaire de la Ville de Paris a mis en place, à destination de l'ensemble de ses agents, un programme baptisé de l'acronyme COCA (Communication, Ouverture, Créativité, Adaptabilité), dans le but de développer des compétences fondamentales comme la rédaction d'un document de synthèse en ayant recours à des logiciels spécialisés (C.N.P.F. Journées internationales de la formation. Deauville 1998);

4. abstraction croissante du travail, le réel cédant progressivement la place au virtuel, et l'hypertexte ou macrotexte remplaçant peu à peu le bon vieux texte ;

5. multiplication des formes de travail : travail à temps partiel, travail partagé, télétravail, travail nomade, missions externes..., qui exigent, de plus en plus, la pluridisciplinarité et la polyvalence, et qui font souvent du professionnel de l'écriture, une sorte d'hommeorchestre ; 
un jour écrivain littéraire, il rédige le lendemain un guide touristique, corrige des épreuves le surlendemain, concocte des accroches publicitaires la semaine suivante, profite de ses déplacements pour effectuer des reportages [...]. (Delteil $2000: 243$ )

Ajoutons, pour conclure, que la logique de compétences rédactionnelles s'inscrit dans une vision, non pas innéiste et abstraite, mais résolument empiriste et pragmatique. Les compétences, en général, et, en rédactologie, en particulier, s'acquièrent, se développent, se multiplient et se renforcent, mais aussi régressent, diminuent, dépérissent et disparaissent, d'où l'importance vitale, dans le processus de leur acquisition et de leur maintenance, de la formation aussi bien initiale que professionnelle continue. On ne naît pas maçon ou traducteur, on le devient.

\section{BIBLIOGRAPHIE}

American Psychological Association (APA). 1990. Publication Manual, Third edition. Washington, D.C.

Beaudet, Céline. 2000. Université Laval. Colloque Rédaction professionnelle et impact social. ACFAS. 23/03/2002. <http://www.usherb.ca/flsh/rrp/no1_beaudet_res.htm>.

Bernard, Claude. 1966. Introduction à l'étude de la médecine expérimentale. Paris : GarnierFlammarion.

Cabré, Maria Teresa. 1998. La terminologie. Canada : Les Presses de l'Université d'Ottawa.

C.N.P.F. 1998. « Nouvelles technologies de l'information et de la communication : Nouvelles compétences ». Objectifs compétences, Tome 11. Paris : CNPF.

Dejean Le Féal, Karla. 1994. « Pédagogie raisonnée de la traduction ». Terminologie et Traduction 3, 7-66. Luxembourg : Office des Publications Officielles des Communautés Européennes.

Deltei, Gérard. 2000. Les métiers de l'écriture. Paris : Rebondir.

De Montmollin, M. 1984. L'intelligence de la tâche - Éléments d'ergonomie cognitive. Beme : Éditions Peter Lang, Science pour la communication.

Durieux, Christine. 1997. «Traduction et linguistique textuelle ». Terminologie et Traduction 1. 49-61. Luxembourg: Office des publications officielles des Communautés européennes.

Durkheim, Émile. 1967. Les règles de la méthode sociologique. Paris : Presses Universitaires de France.

Fromaget, Michel. 1974-1975. « Approche quantitative de la Weltanschauung ». Bulletin de psychologie 319, tome XXVIII- 18.

Gelb, I. J. 1973. Pour une théorie de l'écriture. Paris : Flammarion.

Gilbert, Patrick. 1993. "L'utilisation du concept de compétence en gestion des ressources humaines. Discours et pratiques ». In La psychologie du travail à l'aube du XXI siècle. Issy-LesMoulineaux : Éditions EAP. 
Jakobson, Roman. 1963. Essais de linguistique générale, traduit de l'anglais et préface par Nicolas Ruwet. Paris : Éditions de Minuit.

Ladmiral, Jean-René. 1994. Traduire : théorèmes pour la traduction. Paris : Gallimard.

Lalande, André. 1968. Vocabulaire technique et critique de la philosophie. Paris : Presses Universitaires de France.

Lartigue, Miren. 1999. Le guide des métiers d'avenir. Jeunes Éditions.

Le Boterf, G. 1990. L'ingénierie et l'évaluation de la formation. Paris : Les Éditions d'Organisation.

Leroi-Gourhan, André. 1964. Le geste et la parole. Technique et langage. Paris : Albin Michel.

Magnet, Anne. 2001. « Étude de l'article scientifique de recherche en anglais - Discours sur la méthode ». In Mémet, Monique et Michel Petit. L'anglais de spécialité en France. Bordeaux : GERAS éditeur, 54-71.

Merleau-Ponty, M. 1945. Phénoménologie de la perception. Paris : Gallimard.

Plutarque. 1995. Dialogues. Paris : France Loisirs.

Poincaré, Henri. 1970. La valeur de la science. Paris : Flammarion.

Resche, Catherine. 1999. « Un réseau de voies d'accès à la langue spécialisée en anglais L2 ». ASp 23-26, 349-373.

Schaff, Adam. 1969. Langage et connaissance. Mayenne : Éditions Anthropos.

Ullmo, Jean. 1969. La pensée scientifique moderne. Paris : Flammarion.

\section{RÉSUMÉS}

Une science se définit essentiellement par son objet, son domaine, sa méthode et l'ensemble des savoirs et savoir-faire qu'elle mobilise. La rédactolologie, étude des conduites scripturales, fait partie d'un conglomérat dynamique composé notamment de la terminologie, de la néologie et de la traductologie et formant, en quelque sorte, un noyau actif au centre d'un hyperespace rédactologique. Son champ méthodologique, également complexe, se structure autour de deux dimensions bipolaires fondamentales : la première dimension, relative à la forme du document, s'étend sur un axe "subnormé vs surnormé »; la deuxième dimension, se rapportant au fond, s'étend sur un axe "expérientiel vs expérimental ». Il n'est donc pas surprenant, eu égard à l'étendue de son domaine et à la variété de sa méthode, que la rédactologie requière une nécessaire polyvalence et de très nombreuses compétences, parmi lesquelles l'aptitude à communiquer occupe une position centrale.

A science is essentially defined by its object, domain, method, and all the knowledge and knowhow it summons. The science of writing, as the study of scriptural behaviours, belongs to a dynamic assembly composed, among others, of terminology, neology and the science of translation. It somehow constitutes an active nucleus amidst a writing hyperspace. Its methodological area is equally complex and stretches between two basic poles - the first refers to the form of the document and ranges along a "sub- to super-normed" axis - the second pole refers to the content, and ranges along an "experiential vs experimental" axis. It then comes as no surprise that the science of writing, with its wide scope, the variety of its methods, necessarily requires a polyvalent approach and numerous skills, among which the ability to communicate holds a central position. 
INDEX

Keywords : arch-competence, science of writing, translatology, writing competence, writing method

Mots-clés : archicompétence, compétence rédactionnelle, méthode rédactologique,

rédactologie, traductologie

\section{AUTEUR}

\section{KHÉDIJA NAKBI}

Khédija Nakbi prépare actuellement une thèse portant sur la traduisibilité et les stratégies de traduction des termes spécialisés (anglais-français), sous la direction de Monsieur le Professeur Claude Sionis, Université de Nantes.knakbi@yahoo.fr 